TRANSACTIONS OF THE

AMERICAN MATHEMATICAL SOCIETY

Volume 355, Number 2, Pages 477-492

S 0002-9947(02)03091-X

Article electronically published on August 27, 2002

\title{
THE CO-AREA FORMULA FOR SOBOLEV MAPPINGS
}

\author{
JAN MALÝ, DAVID SWANSON, AND WILLIAM P. ZIEMER
}

\begin{abstract}
We extend Federer's co-area formula to mappings $f$ belonging to the Sobolev class $W^{1, p}\left(\mathbb{R}^{n} ; \mathbb{R}^{m}\right), 1 \leq m<n, p>m$, and more generally, to mappings with gradient in the Lorentz space $L^{m, 1}\left(\mathbb{R}^{n}\right)$. This is accomplished by showing that the graph of $f$ in $\mathbb{R}^{n+m}$ is a Hausdorff $n$-rectifiable set.
\end{abstract}

\section{INTRODUCTION}

The following equality, first proved by Federer in [F4 and termed the co-area formula, has proved to be a fundamental tool in analysis with a wide variety of applications:

$$
\int_{\Omega} g(x)\left|J_{m} f(x)\right| d x=\int_{\mathbb{R}^{m}} \int_{f^{-1}(y)} g(x) d \mathcal{H}^{n-m}(x) d y,
$$

where $\Omega \subset \mathbb{R}^{n}$ is an open set, $f: \Omega \rightarrow \mathbb{R}^{m}$ is Lipschitz, $J_{m} f$ is its $m$-dimensional Jacobian, $g: \Omega \rightarrow \mathbb{R}$ is integrable, and $1 \leq m<n$. Recall that $\left|J_{m} f\right|$ is the square root of the sum of the squares of the determinants of the $m$ by $m$ minors of the differential of $f$. Using standard approximation methods, (1.1) may be obtained from the special case where $g$ is the indicator of a measurable set $E \subset \mathbb{R}^{n}$ :

$$
\int_{E}\left|J_{m} f(x)\right| d x=\int_{\mathbb{R}^{m}} \mathcal{H}^{n-m}\left(E \cap f^{-1}(y)\right) d y .
$$

Because of the usefulness of the co-area formula, a natural and compelling question is whether it will admit an extension to a larger class of mappings, in particular, to the class $W_{\text {loc }}^{1,1}\left(\Omega ; \mathbb{R}^{m}\right)$ of Sobolev mappings. When $m=1$, it was shown by Federer [F5] that (1.2) is valid for mappings $f \in W^{1, p}(\Omega), p \geq 1$, provided that $f$ is precisely represented; see (2.5) below. The case $p>n$, including $m \geq 2$, has been considered by Van der Putten VP.

One of the main objectives of this paper is to establish (1.2) for mappings $f$ belonging to the Sobolev class $W_{\text {loc }}^{1, p}\left(\Omega ; \mathbb{R}^{m}\right)$. The following result is new for $m>1$ and the proof simplifies the existing proof for $m=1$.

Received by the editors December 3, 2001

2000 Mathematics Subject Classification. Primary 46E35, 46E30; Secondary 26B10, 26B35, 49Q15.

Key words and phrases. Sobolev mapping, Orlicz space, co-area formula, area formula, rectifiability.

The research of the first author is supported in part by the Research Project MSM 113200007 from the Czech Ministry of Education, Grant No. 201/00/0767 from the Grant Agency of the Czech Republic (GA ČR) and Grant No. 165/99 from the Grant Agency of Charles University (GA UK). 
1.1. Theorem. Suppose that $1 \leq m \leq n$ and that $f \in W_{\text {loc }}^{1, p}\left(\Omega ; \mathbb{R}^{m}\right)$ is precisely represented, where either $p>m$ or $p \geq m=1$. Then $f^{-1}(y)$ is countably $\mathcal{H}^{n-m}$ rectifiable for almost all $y \in \mathbb{R}^{m}$ and the co-area formula (1.2) holds for all measurable sets $E \subset \Omega$.

In the following theorem, we denote by $\bar{f}: \Omega \rightarrow \mathbb{R}^{n+m}$ the graph mapping $\bar{f}(x)=(x, f(x))$ and by $\mathcal{G}_{f}$ the graph of $f: \mathcal{G}_{f}=\bar{f}(\Omega) \subset \mathbb{R}^{n+m}$.

1.2. Theorem. Suppose that $1 \leq m \leq n$ and that $f \in W_{\text {loc }}^{1, p}\left(\Omega ; \mathbb{R}^{m}\right)$ is precisely represented, where either $p>m$ or $p \geq m=1$. Then $\mathcal{G}_{f}$ is countably $\mathcal{H}^{n}$ rectifiable and

$$
\mathcal{H}^{n}(\bar{f}(E))=\int_{E}\left|J_{n} \bar{f}(x)\right| d x
$$

for all measurable sets $E \subset \Omega$. In particular, $\mathcal{H}^{n}(\bar{f}(E))=0$ whenever $\mathcal{L}^{n}(E)=0$.

The hypothesis $p>m$ is necessary. In [C], [MM], an example of a continuous mapping $f \in W^{1, m}\left(\mathbb{R}^{m} ; \mathbb{R}^{m}\right)$ is exhibited that has an almost everywhere vanishing Jacobian and maps an interval $I$ onto an $m$-cube. This may be easily modified in the case $m \geq 2$ to an example of a continuous mapping $f \in W_{\text {loc }}^{1, m}\left(\mathbb{R}^{n} ; \mathbb{R}^{m}\right)$ that has an almost everywhere vanishing $m$-Jacobian and that maps each set of the form $I \times$ $\mathbb{R}^{n-m}$ onto an $m$-cube (cf. [H]), therefore violating the conclusions of Theorems 1.1 and 1.2 However, if we turn our attention to mappings whose gradients belong to the finer scale of Lorentz spaces, we obtain the following extension of the above stated theorems.

1.3. Theorem. Suppose that $1 \leq m \leq n$, that $f \in W_{\text {loc }}^{1,1}\left(\Omega ; \mathbb{R}^{m}\right)$ is precisely represented, and that $|\nabla f| \in L^{m, 1}(\Omega)$. Then $f^{-1}(y)$ is countably $\mathcal{H}^{n-m}$ rectifiable for almost all $y \in \mathbb{R}^{m}$, the graph $\mathcal{G}_{f}$ is countably $\mathcal{H}^{n}$ rectifiable and the co-area formula (1.2) and area formula (1.3) hold for all measurable sets $E \subset \Omega$.

The case $m=n$ of Theorem 1.3 (and even the area counterpart $m \geq n$ ) follows from the work of Kauhanen, Koskela, and Malý [KKM] who generalized the area formula of Marcus and Mizel MMi] to functions whose gradients lie in $L^{n, 1}(\Omega)$.

It is also possible to obtain results dealing with the borderline case $p=m$ for mappings that are Hölder continuous. The following theorem provides a coarea counterpart to the results in $[\mathrm{MM}]$ on the area formula for Hölder continuous mappings in $W^{1, n}$. (See also the recent developments in [M2], [FM], M4].)

1.4. Theorem. Suppose that $1 \leq m \leq n$ and that $f \in W^{1, m}\left(\Omega ; \mathbb{R}^{m}\right)$ is a Hölder continuous mapping. Then $f^{-1}(y)$ is countably $\mathcal{H}^{n-m}$ rectifiable for almost all $y \in \mathbb{R}^{m}$ and

$$
\int_{E}\left|J_{m} f(x)\right| d x=\int_{\mathbb{R}^{m}} \mathcal{H}^{n-m}\left(E \cap f^{-1}(y)\right) d y
$$

for all measurable sets $E \subset \Omega$.

Theorem [1.4 is proven independently of any area estimates on the $\mathcal{G}_{f}$ and, in fact, the proof can be modified to give an independent proof of Theorem 1.1 in the case $p>m$; cf. Remark 7.2 below.

Weaker variants of the co-area and area formulas use the integral-geometric measure $\mathcal{I}^{q}$ instead of the Hausdorff measure $\mathcal{H}^{q}$. Hajłasz $[\mathrm{H}]$ proved such a version 
of the co-area formula for $W^{1, p}$-mappings $(p>m)$. We present a version of Theorem 1.2 in the borderline case $p=m$ under the assumption of Hölder continuity. We have not been able to obtain such a result with the Hausdorff measure.

1.5. Theorem. Suppose that $1 \leq m \leq n$ and that $f \in W^{1, m}\left(\Omega ; \mathbb{R}^{m}\right)$ is a Hölder continuous mapping. Then $\mathcal{G}_{f}$ is countably $\mathcal{I}^{n}$ rectifiable and

$$
\mathcal{I}^{n}(\bar{f}(E))=\int_{E}\left|J_{n} \bar{f}(x)\right| d x
$$

for all measurable sets $E \subset \Omega$.

The results listed above are easily localized from $\mathbb{R}^{n}$ to $\Omega$. For the proofs, we may assume that $\Omega=\mathbb{R}^{n}$ and that the global norms of the considered mappings are finite. Since our results follow from the area formulas in [MMi] and [KKM] when $m=n$, we also assume throughout that $m<n$.

\section{Preliminaries}

We denote by $\mathcal{L}^{n}(E)$ the Lebesgue measure of a set $E \subset \mathbb{R}^{n}$ and by $\mathcal{H}^{q}(E)$ the $q$-dimensional Hausdorff measure of $E$. If $q$ is an integer, then $E$ is said to be countably $\mathcal{H}^{q}$ rectifiable if there exist subsets $E_{k} \subset \mathbb{R}^{q}$ and Lipschitz mappings $g_{k}: E_{k} \rightarrow \mathbb{R}^{n}$ with the property that

$$
\mathcal{H}^{q}\left(E \backslash \bigcup_{k=1}^{\infty} g_{k}\left(E_{k}\right)\right)=0 .
$$

The $q$-dimensional Hausdorff content $\mathcal{H}_{\infty}^{q}(E)$ of $E$ is the infimum of the sums

$$
\sum_{j=1}^{\infty} \boldsymbol{\alpha}_{q}\left(\frac{\operatorname{diam} E_{j}}{2}\right)^{q}
$$

corresponding to all countable coverings of $E$ by sets $\left\{E_{j}\right\}_{j=1}^{\infty}$. Here

$$
\boldsymbol{\alpha}_{q}=\frac{\pi^{q / 2}}{\Gamma\left(\frac{q}{2}+1\right)}
$$

which is the volume of the unit ball in $\mathbb{R}^{q}$ if $q$ is an integer. Observe that $\mathcal{H}^{q}(E)=0$ if and only if $\mathcal{H}_{\infty}^{q}(E)=0$. The integral average of a function $g$ over the set $E$ is defined as

$$
f_{E} g(y) d y=\frac{1}{\mathcal{L}^{n}(E)} \int_{E} g(y) d y .
$$

Given $1 \leq p \leq \infty$, the Sobolev space $W^{1, p}(\Omega)$ consists of those functions $f \in$ $L^{p}(\Omega)$ with the property that the distributional gradient $D f$ of $f$ may be identified with a function $\nabla f \in L^{p}\left(\Omega ; \mathbb{R}^{n}\right) . W^{1, p}(\Omega)$ is a Banach space with respect to the norm

$$
\|f\|_{1, p, \Omega}:=\|f\|_{L^{p}(\Omega)}+\|\nabla f\|_{L^{p}(\Omega)} .
$$

The class $W^{1, p}\left(\Omega ; \mathbb{R}^{m}\right), m \geq 1$, consists of those mappings $f: \Omega \rightarrow \mathbb{R}^{m}$ whose component functions each belong to $W^{1, p}(\Omega)$. Qualitative properties like Lebesgue points, approximate differentiability, etc. may be investigated componentwise. 
We will also consider a refined scale of Sobolev spaces consisting of those functions with gradients in Lorentz and Orlicz spaces. If $g$ is a measurable function on $\Omega$, we define the distribution function of $g$ as

$$
\mu_{g}(s)=\mathcal{L}^{n}(\{x \in \Omega:|g(x)|>s\}) .
$$

We set

$$
\|g\|_{L^{m, 1}(\Omega)}=\int_{0}^{\infty} \mu_{g}(s)^{1 / m} d s .
$$

We say that $g$ belongs to the Lorentz space $L^{m, 1}(\Omega)$ if $\|g\|_{L^{m, 1}(\Omega)}<\infty$.

It is known that this space can be expressed as a union of Orlicz spaces. A function $\mathbf{F}:[0,+\infty) \rightarrow \mathbb{R}$ is said to be a Young function if $\mathbf{F}$ is convex, nonnegative and satisfies

$$
\mathbf{F}(t)=0 \Longleftrightarrow t=0
$$

The Orlicz space $L^{\mathbf{F}}(\Omega)$ is then defined as the space of all measurable functions $g$ on $\Omega$ for which there exists $\lambda>0$ such that

$$
\int_{\Omega} \mathbf{F}(|g| / \lambda) d x \leq 1
$$

The following result is proven in [MSZ].

2.1. Proposition. Let $g \in L^{m, 1}(\Omega)$ and $1<q<m<p$. Then there is a $\mathcal{C}^{1}$ Young function $\mathbf{F}$ and a constant $C=C(m, p, q)>0$ such that

$$
\begin{gathered}
\int_{\Omega} \mathbf{F}(|g|) d x \leq C\|g\|_{L^{m, 1}(\Omega)}^{m}, \\
\int_{0}^{\infty} \mathbf{F}^{\prime}(t)^{-\frac{1}{m-1}} d t \leq C,
\end{gathered}
$$

and

$$
q \leq \frac{t \mathbf{F}^{\prime}(t)}{\mathbf{F}(t)} \leq p, \quad t>0 .
$$

If a Young function $\mathbf{F}$ satisfies (2.3), then the function $t^{p} \mathbf{F}(t)$ is nondecreasing and the function $t^{q} \mathbf{F}(t)$ is nonincreasing. In this case, the Orlicz space $L^{\mathbf{F}}(\Omega)$ coincides with the set of all measurable functions for which the integral $\int_{\Omega} \mathbf{F}(|g|) d x$ converges.

In the following paragraphs, we recall the definition of capacity and certain pointwise properties of Sobolev functions. We refer the reader to FZ for the proofs.

For $1 \leq p<\infty$, the $p$-capacity $\gamma_{p}(E)$ of a set $E \subset \mathbb{R}^{n}$ is the infimum of numbers of the form

$$
\int_{\mathbb{R}^{n}}\left(|u|^{p}+|\nabla u|^{p}\right) d x
$$

corresponding to all $u \in W^{1, p}\left(\mathbb{R}^{n}\right)$ with the property that $u \geq 1$ on a neighborhood of $E$. $p$-Capacity is related to Hausdorff measure as follows: if $p>1$ and $\gamma_{p}(E)=0$, then $\mathcal{H}^{q}(E)=0$ for all $q>n-p$, whereas $\mathcal{H}^{n-p}(E)<\infty$ implies $\gamma_{p}(E)=0$. As for the case $p=1$, Fleming [F] proved that $\gamma_{1}(E)=0$ if and only if $\mathcal{H}^{n-1}(E)=0$. Related statements concerning the Hausdorff content are given in Lemma 5.1 and Theorem 5.5 below. 
A function $f: \Omega \rightarrow \mathbb{R}^{n}$ is said to be $p$-quasicontinuous if for every $\varepsilon>0$, there exists an open set $G$ with $\gamma_{p}(G)<\varepsilon$ so that $\left.f\right|_{\Omega \backslash G}$ is continuous. Any two $p$ quasicontinuous functions that agree almost everywhere agree up to a set $E$ with $\gamma_{p}(E)=0$.

If $\Omega \subset \mathbb{R}^{n}$ is an open set and $f \in L_{\text {loc }}^{1}(\Omega)$, then a function $\tilde{f}$ is said to be a precise representative of $f$ if

$$
\tilde{f}(x):=\lim _{r \rightarrow 0} f_{B(x, r)} f(y) d y
$$

at all points $x$ where this limit exists. It is clear from the Lebesgue differentiation theorem that any function $f \in L_{\text {loc }}^{1}(\Omega)$ may be modified on a set of Lebesgue measure zero so as to be precisely represented and that any two precise representatives coincide almost everywhere. A mapping $f \in L_{\text {loc }}^{1}\left(\Omega ; \mathbb{R}^{m}\right)$ is said to be precisely represented if each of its component functions is a precise representative.

Fundamental properties of functions $f \in W^{1, p}(\Omega)$ are that the limit (2.5) exists for all $x$ outside a set $E$ with $\gamma_{p}(E)=0$, and that any precise representative of a function $f \in W^{1, p}(\Omega)$ is $p$-quasicontinuous. Thus if $p>m$ or $p=m=1$, any two precise representatives agree outside a set $E$ with $\mathcal{H}^{n-m}(E)=0$. Since sets of $\mathcal{H}^{n-m}$ measure zero are negligible for results of Theorem [1.1 and Theorem 1.2. it follows that their statements will hold for any $p$-quasicontinuous representative and even any representative with Lebesgue points $\mathcal{H}^{n-m}$ almost everywhere.

Similarly it is proven in [MSZ that if $f \in W_{\text {loc }}^{1,1}(\Omega)$ and $|\nabla f| \in L^{m, 1}(\Omega)$, then any precise representative of $f$ is defined up to a set $E$ with $\mathcal{H}^{n-m}(E)=0$. It follows that the statement of Theorem 1.3 will hold for any representative with Lebesgue points $\mathcal{H}^{n-m}$ almost everywhere.

\section{Lusin's Condition (N)}

A mapping $f: \mathbb{R}^{n} \rightarrow \mathbb{R}^{k}, k \geq n$, is said to satisfy Lusin's condition (N) if $\mathcal{H}^{n}(f(E))=0$ whenever $E \subset \mathbb{R}^{n}$ satisfies $\mathcal{L}^{n}(E)=0$. In this section, we show that if $f \in W^{1, p}\left(\mathbb{R}^{n} ; \mathbb{R}^{m}\right)$ and $\bar{f}: \mathbb{R}^{n} \rightarrow \mathbb{R}^{n+m}$ satisfies condition (N), then the conclusions of Theorems 1.1 and 1.2 hold. This holds without any particular restriction on $p$. First we require two well-known lemmas.

3.1. Lemma (Eilenberg inequality, [E]). Suppose $m \leq d \leq m+n, A \subset \mathbb{R}^{n+m}$, and $h: A \rightarrow \mathbb{R}^{m}$ is Lipschitz. Then

$$
\int^{*} \mathcal{H}^{d-m}\left(A \cap h^{-1}(y)\right) d \mathcal{H}^{m}(y) \leq C(\operatorname{Lip} h)^{m} \mathcal{H}^{d}(A),
$$

where $\int^{*}$ denotes the upper integral, Lip $f$ is the Lipschitz constant of $f$, and $C=$ $C(m)$ is a constant depending only on $m$.

3.2. Lemma. If $f \in W_{\text {loc }}^{1,1}\left(\mathbb{R}^{n} ; \mathbb{R}^{m}\right)$, then there exist Lipschitz functions $f_{k}: \mathbb{R}^{n} \rightarrow$ $\mathbb{R}^{m}$ and disjoint subsets $E_{k}$ of $\mathbb{R}^{n}$ such that $f=f_{k}$ on $E_{k}$ and $\mathcal{L}^{n}\left(\mathbb{R}^{n} \backslash \bigcup E_{k}\right)=0$.

This follows from the a.e. approximate differentiability of $f$ [F5, 3.1.4], [BZ] and a general property of a.e. approximately differentiable functions [F5, 3.1.8].

3.3. Theorem. Let $f \in W_{\mathrm{loc}}^{1,1}\left(\mathbb{R}^{n} ; \mathbb{R}^{m}\right), 1 \leq m \leq n$, and suppose that $\bar{f}$ satisfies condition $(N)$. Then $f^{-1}(y)$ is countably $\mathcal{H}^{n-m}$ rectifiable for almost all $y \in \mathbb{R}^{m}$, the graph $\mathcal{G}_{f}$ is countably $\mathcal{H}^{n}$ rectifiable and the co-area formula (1.2) and area formula (1.3) hold for all measurable sets $E \subset \Omega$. 
Proof. Choose functions $f_{k}$ and sets $E_{k}$ as in Lemma 3.2. Since $f_{k}=f$ on $E_{k}$ and $\nabla f_{k}=\nabla f$ a.e. on $E_{k}$, it follows from the classical area formula for Lipschitz functions [F5, 3.2.5] that

$$
\mathcal{H}^{n}(\bar{f}(E))=\int_{E}\left|J_{n} \bar{f}(x)\right| d x
$$

whenever $E \subset E_{k}$. Moreover, (3.1) will be satisfied whenever $\mathcal{L}^{n}(E)=0$ provided that $\bar{f}$ satisfies condition $(\mathrm{N})$. Therefore, the monotone convergence theorem implies (3.1) for all $E \subset \mathbb{R}^{n}$, as desired. That the graph $\mathcal{G}_{f}$ is countably $\mathcal{H}^{n}$ rectifiable is evident since

$$
\mathcal{H}^{n}\left(\mathcal{G}_{f} \backslash \bigcup_{k=1}^{\infty} \bar{f}\left(E_{k}\right)\right)=0 .
$$

Now denote by $\pi: \mathbb{R}^{n+m} \rightarrow \mathbb{R}^{n}$ and $\rho: \mathbb{R}^{n+m} \rightarrow \mathbb{R}^{m}$ the projections

$\pi\left(x, x_{n+1}, \ldots, x_{n+m}\right)=x \quad$ and $\quad \rho\left(x, x_{n+1}, \ldots, x_{n+m}\right)=\left(x_{n+1}, \ldots, x_{n+m}\right)$.

Let $E \subset \mathbb{R}^{n}$ satisfy $\mathcal{L}^{n}(E)=0$ and apply the Eilenberg inequality with $A=\bar{f}(E)$, $h=\rho$, and $d=n$. Then

$$
\int_{\mathbb{R}^{m}} \mathcal{H}^{n-m}\left(A \cap \rho^{-1}(y)\right) d \mathcal{H}^{m}(y) \leq C \mathcal{H}^{n}(\bar{f}(E))=0 .
$$

Now, $A \cap \rho^{-1}(y)=\left\{(x, f(x)) \in \mathbb{R}^{n+m}: x \in E, f(x)=y\right\}$; so it follows that $\pi\left(A \cap \rho^{-1}(y)\right)=E \cap f^{-1}(y)$. Since Hausdorff measures do not increase on projection, we conclude

$$
\int_{\mathbb{R}^{m}} \mathcal{H}^{n-m}\left(E \cap f^{-1}(y)\right) d \mathcal{H}^{m}(y)=0 .
$$

From (1.2) above, we have that

$$
\int_{\mathbb{R}^{m}} \mathcal{H}^{n-m}\left(E \cap f^{-1}(y)\right) d \mathcal{H}^{m}(y)=\int_{E}\left|J_{m} f(x)\right| d x
$$

holds whenever $E \subset E_{k}$; so again the monotone convergence theorem implies that (3.1) holds for all $E \subset \mathbb{R}^{n}$. That $f^{-1}(y)$ is countably $\mathcal{H}^{n-m}$ rectifiable for almost all $y \in \mathbb{R}^{m}$ follows from the fact that for each $k, E_{k} \cap f^{-1}(y)=E_{k} \cap f_{k}^{-1}(y)$ is countably $\mathcal{H}^{n-m}$ rectifiable for almost all $y \in \mathbb{R}^{m}$ (see [F5, 3.2.15]), and that $\mathcal{H}^{n-m}\left(E \cap f^{-1}(y)\right)=0$ for almost all $y$ whenever $\mathcal{L}^{n}(E)=0$.

\section{A general CRiterion for COndition $N$}

Throughout this section we denote by $\pi: \mathbb{R}^{n+m} \rightarrow \mathbb{R}^{n}$ the projection

$$
\pi\left(x, x_{n+1}, \ldots, x_{n+m}\right)=x .
$$

4.1. Lemma. Suppose $m \leq d \leq m+n$. Let $E \subset \mathbb{R}^{n+m}$. Then

$$
\mathcal{H}_{\infty}^{d}(E) \leq C(\operatorname{diam} E)^{m} \mathcal{H}_{\infty}^{d-m}(\pi(E)),
$$

where $C=C(m, n, d)$.

Proof. Assume diam $E<\infty$. Cover $\pi(E)$ with a sequence of sets $\left\{A_{k}\right\}_{k=1}^{\infty}$ of finite and positive diameter. For each $k$, let $N_{k}$ denote the greatest integer satisfying

$$
\left(N_{k}-1\right) \cdot\left(\operatorname{diam} A_{k}\right)<\operatorname{diam} E .
$$


The set $E \cap \pi^{-1}\left(A_{k}\right)$ is easily seen to be contained in a cylinder of the form $A_{k} \times Q_{k}$, where $Q_{k}$ is a cube in $\mathbb{R}^{m}$ with side-length $\operatorname{diam} E$. Since $N_{k} \cdot\left(\operatorname{diam} A_{k}\right) \geq \operatorname{diam} E$, $Q_{k}$ may be covered by $N_{k}^{m}$ cubes $\left\{Q_{k}^{j}\right\}$ of side-length $\operatorname{diam} A_{k}$. It follows that

$$
\begin{aligned}
\mathcal{H}_{\infty}^{d}\left(E \cap \pi^{-1}\left(A_{k}\right)\right) & \leq C \sum_{j=1}^{N_{k}^{m}}\left(\frac{\operatorname{diam}\left(A_{k} \times Q_{k}^{j}\right)}{2}\right)^{d} \\
& \leq C N_{k}^{m} \cdot\left(\operatorname{diam} A_{k}\right)^{d} \\
& \leq C\left(\operatorname{diam} A_{k}+\operatorname{diam} E\right)^{m} \cdot\left(\operatorname{diam} A_{k}\right)^{d-m} .
\end{aligned}
$$

Since $\operatorname{diam} A_{k} \leq \operatorname{diam} \pi(E) \leq \operatorname{diam} E$, summing over $k$ shows that

$$
\mathcal{H}_{\infty}^{d}(E) \leq C(\operatorname{diam} E)^{m} \sum_{k=1}^{\infty}\left(\operatorname{diam} A_{k}\right)^{d-m} .
$$

The result follows by taking the infimum over all coverings $\left\{A_{k}\right\}$.

4.2. Corollary. Suppose $m \leq d \leq m+n$. Let $E \subset \mathbb{R}^{n+m}$ and $\mathcal{H}^{d-m}(\pi(E))=0$. Then $\mathcal{H}^{d}(E)=0$.

The following lemma concerns a measurability property of the graph of a mapping $f$ and is used in the proof of Theorem 4.4 below. Recall that $\bar{f}$ denotes the graph mapping $\bar{f}(x)=(x, f(x))$ and $\mathcal{G}_{f}$ the graph set $\bar{f}\left(\mathbb{R}^{n}\right) \subset \mathbb{R}^{n+m}$.

4.3. Lemma. Let $f: \mathbb{R}^{n} \rightarrow \mathbb{R}^{m}$ be an $\mathcal{L}^{n}$ measurable mapping. Then $\pi\left(\mathcal{G}_{f} \cap E\right)$ is $\mathcal{L}^{n}$ measurable for every Borel set $E \subset \mathbb{R}^{n+m}$.

Proof. Let $f^{*}$ be a Borel measurable representative of $f$. The graph $\mathcal{G}_{f^{*}}$ of such a mapping is a Borel subset of $\mathbb{R}^{n+m}$, implying that the projection $\pi\left(\mathcal{G}_{f^{*}} \cap E\right)$ is a Borel subset of $\mathbb{R}^{n}$ for any Borel set $E \subset \mathbb{R}^{n+m}$. See $[\mathrm{K}, \S 31$. VII]. Since $f$ and $f^{*}$ agree up to a set of $\mathcal{L}^{n}$ measure zero, so do the sets $\pi\left(\mathcal{G}_{f^{*}} \cap E\right)$ and $\pi\left(\mathcal{G}_{f} \cap E\right)$. Thus $\pi\left(\mathcal{G}_{f} \cap E\right)$ is $\mathcal{L}^{n}$ measurable.

The following result yields a criterion similar to that of Radó and Reichelderfer; see $[\mathrm{RR}]$, $\mathrm{M} 3$.

4.4. Theorem. Suppose that $1 \leq m \leq n$. Let $f: \mathbb{R}^{n} \rightarrow \mathbb{R}^{m}$ be measurable and suppose that there is $\theta \in L_{\text {loc }}^{1}\left(\mathbb{R}^{n}\right)$ such that

$$
\mathcal{H}_{\infty}^{n-m}\left(\pi\left(\mathcal{G}_{f} \cap B(z, r)\right)\right) \leq r^{-m} \int_{\pi\left(\mathcal{G}_{f} \cap B(z, 4 r)\right)} \theta(x) d x
$$

for all $z \in \mathbb{R}^{n+m}$ and $r>0$. Then there is a constant $C=C(m, n)$ depending only on $m$ and $n$ so that

$$
\mathcal{H}^{n}(\bar{f}(E)) \leq C \int_{E} \theta(x) d x
$$

for all $\mathcal{L}^{n}$ measurable $E \subset \mathbb{R}^{n}$. In particular, $\bar{f}$ satisfies condition $(N)$.

Proof. Define a set function $\sigma$ on $\mathbb{R}^{n+m}$ by

$$
\sigma(E)=\int_{\pi\left(\mathcal{G}_{f} \cap E\right)} \theta(x) d x .
$$


Lemma 4.1 with $d=n$ and (4.1) imply that

$$
\begin{aligned}
\mathcal{H}_{\infty}^{n}\left(\mathcal{G}_{f} \cap B(z, r)\right) & \leq C r^{m} \mathcal{H}_{\infty}^{n-m}\left(\pi\left(\mathcal{G}_{f} \cap B(z, r)\right)\right) \\
& \leq C \int_{\pi\left(\mathcal{G}_{f} \cap B(z, 4 r)\right)} \theta(x) d x \\
& =C \sigma(B(z, 4 r))
\end{aligned}
$$

for any $z \in \mathbb{R}^{n+m}$ and $r>0$. Using the fact that

$$
\limsup _{r \rightarrow 0^{+}} r^{-n} \mathcal{H}_{\infty}^{n}\left(\mathcal{G}_{f} \cap B(z, r)\right) \geq C
$$

for $\mathcal{H}^{n}$ almost every $z \in \mathcal{G}_{f}$ ([F2, Lemma 10.1]), it follows that

$$
\limsup _{r \rightarrow 0^{+}} r^{-n} \sigma(B(z, r)) \geq C
$$

for $\mathcal{H}^{n}$ almost all $z \in \mathcal{G}_{f}$. Now, Lemma 4.3 implies that $\sigma$ is a measure on the Borel $\Sigma$-algebra of $\mathbb{R}^{n+m}$, and so it may be extended to a regular Borel (outer) measure $\sigma^{*}$ on all of $\mathbb{R}^{n+m}$ in the usual way:

$$
\sigma^{*}(E):=\inf \{\sigma(B): E \subset B, B \text { a Borel set }\} .
$$

Since $\theta$ is locally integrable, it follows that $\sigma^{*}$ is a Radon measure on $\mathbb{R}^{n+m}$. Therefore [F2, Theorem 10.3], [M] Theorem 6.9], and (4.2) imply that $\mathcal{H}^{n}(E) \leq C \sigma^{*}(E)$ for all $E \subset \mathcal{G}_{f}$. Finally, given an $\mathcal{L}^{n}$ measurable set $E \subset \mathbb{R}^{n}$, choose a Borel set $G$ with $E \subset G$. Then $\bar{f}(E) \subset G \times \mathbb{R}^{m}, G \times \mathbb{R}^{m}$ is Borel, and

$$
\mathcal{H}^{n}(\bar{f}(E)) \leq C \sigma^{*}(\bar{f}(E)) \leq C \sigma\left(G \times \mathbb{R}^{m}\right)=C \int_{G} \theta(x) d x .
$$

The proof is completed by taking the infimum over all such $G$.

\section{Capacitary estimates}

The next lemma gives a capacitary estimate for $W^{1,1}$ which relates the 1-capacity with the $(n-1)$-dimensional Hausdorff content. This result is due to Fleming [Fl], whose proof depends on information based on BV functions, in particular, sets of finite perimeter. We give an independent proof which circumvents the need for BV theory.

5.1. Lemma. Let $E \subset \mathbb{R}^{n}$. Then $\mathcal{H}_{\infty}^{n-1}(E) \leq C \gamma_{1}(E)$, where $C=C(n)$.

Proof. Let $u$ be a test function for the capacity. Let us consider the continuous monotone real function

$$
\psi(t)=\int_{\{0<u<t\}}(|u|+|\nabla u|) d y .
$$

Then $\psi$ is a.e. differentiable and

$$
\int_{0}^{1} \psi^{\prime}(t) d t \leq \psi(1)-\psi(0)=\psi(1) .
$$

Hence there exists $s \in(0, \ell)$ such that $\psi^{\prime}(s)<2 \psi(1)$. We find $\delta>0$ such that

$$
\frac{\psi\left(s^{\prime}\right)-\psi(s)}{s^{\prime}-s} \leq 2 \psi(1) \quad \text { for each } s^{\prime} \in(s, s+\delta) .
$$

Let $x \in E$. Then $u(x) \geq 1$ in a neighborhood of $x$ and so the set

$$
R_{x}:=\left\{\rho:|B(x, \rho) \cap\{u \geq s\}|<\frac{1}{2}|B(x, \rho)|\right\}
$$


is nonempty. Let us define

$$
r_{x}=\sup R_{x}
$$

Then obviously $\sup _{x \in E} r_{x}<\infty$ and

$$
\left|B\left(x, r_{x}\right) \cap\{u<s\}\right| \leq \frac{1}{2}\left|B\left(x, r_{x}\right)\right|
$$

and

$$
\left|B\left(x, r_{x}\right) \cap\{u \geq s\}\right| \leq \frac{1}{2}\left|B\left(x, r_{x}\right)\right| .
$$

We use the Besicovitch covering theorem to extract a (finite or infinite) sequence $\left\{B_{j}\right\}_{j \in I}$ of balls $B_{j}=B\left(x_{j}, r_{j}\right)$ from $\left\{B\left(x, r_{x}\right): x \in E\right\}$ such that it covers the set $E$ and its overlap multiplicity is bounded by an integer $M$ depending only on $n$. Here $I=\mathbb{N}$ or $I=\left\{1,2, \ldots, i_{\max }\right\}$. Fix $i \in I$. Using (5.3), we find a level $t_{i} \in(s, s+\delta)$ such that

$$
\left|B\left(x_{j}, r_{j}\right) \cap\left\{u \leq t_{i}\right\}\right| \leq \frac{3}{4}\left|B\left(x_{j}, r_{j}\right)\right|, \quad j=1, \ldots, i .
$$

Consider the truncated function

$$
u_{i}= \begin{cases}t_{i} & \text { on }\left\{u \geq t_{i}\right\}, \\ s & \text { on }\{u \leq s\}, \\ u & \text { on }\left\{s<u<t_{i}\right\} .\end{cases}
$$

Let $s_{i, j}$ be a median value of $u_{i}$ on $B_{j}$. From (5.2) and (5.4), we infer that

$$
t_{i}-s \leq C f_{B_{j}}\left|u_{i}-s_{i, j}\right| d y
$$

By the Poincaré inequality we can continue

$$
t_{i}-s \leq C r_{j} f_{B_{j}}\left|\nabla u_{i}\right| d y
$$

This means that

$$
r_{j}^{n-1} \leq \frac{C}{t_{i}-s} \int_{B_{j}}\left(\left|u_{i}\right|+\left|\nabla u_{i}\right|\right) d y .
$$

Summing over $j=1, \ldots, i$ and using (5.1), we obtain

$$
\begin{aligned}
\sum_{j=1}^{i} r_{j}^{n-1} & \leq \frac{C}{t_{i}-s} \sum_{j=1}^{i} \int_{B_{j}}\left(\left|u_{i}\right|+\left|\nabla u_{i}\right|\right) d y \leq \frac{C M}{t_{i}-s} \int_{B}\left(\left|u_{i}\right|+\left|\nabla u_{i}\right|\right) d y \\
& =\frac{C M}{t_{i}-s} \int_{B \cap\left\{s \leq u<t_{i}\right\}}(|u|+|\nabla u|) d y=C M \frac{\psi\left(t_{i}\right)-\psi(s)}{t_{i}-s} \\
& \leq C \psi(1) M .
\end{aligned}
$$

We can pass with $i$ to $i_{\max }$ or $\infty$ and we obtain

$$
\mathcal{H}_{\infty}^{n-1}(E) \leq C \psi(1) \leq \int_{\mathbb{R}^{n}}(|u|+|\nabla u|) d y
$$

as required.

5.2. Corollary. Let $E \subset \mathbb{R}^{n}$. Suppose that $u \in W^{1,1}\left(\mathbb{R}^{n}\right)$ is precisely represented and that $u \geq 1$ on $E$. Then

$$
\mathcal{H}_{\infty}^{n-1}(E) \leq C \int_{\mathbb{R}^{n}}(|\nabla u|+|u|) d x
$$


Proof. Any precise representative of $u \in W^{1,1}\left(\mathbb{R}^{n}\right)$ has Lebesgue points $\mathcal{H}^{n-1}$-a.e., cf. [Fl, [FZ]. Hence we may assume that $u$ has a Lebesgue point at $\mathcal{H}^{n-1}$-a.e. point of $x \in E$. Given $\varepsilon>0$, let $\left\{u_{k}\right\}$ be a sequence of smooth functions approximating $u$ and obtained by mollification, such that

$$
\left\|u_{k}-u\right\|_{1,1} \leq 2^{-k-1} \varepsilon
$$

Set

$$
w=u_{1}+\sum_{k=1}^{\infty}\left|u_{k+1}-u_{k}\right|
$$

Then $w \in W^{1,1}\left(\mathbb{R}^{n}\right),\|w\|_{1,1} \leq\|u\|_{1,1}+\varepsilon$ and $w \geq 1$ on a neighborhood of $E$. Hence,

$$
\mathcal{H}_{\infty}^{n-1}(E) \leq C \int_{\mathbb{R}^{n}}(|\nabla w|+|w|) d x \leq C \int_{\mathbb{R}^{n}}(|\nabla u|+|u|) d x+C \varepsilon .
$$

Letting $\varepsilon \rightarrow 0$, we obtain the assertion.

For the proofs of Theorems 1.1 and 1.2 we need the well-known relation between the $p$-capacity and the Hausdorff content; see, e.g., [AH] Theorem 5.1.13]. This result goes back to Frostman $(p=2)$ and the case of general $p$ is due to Reshetnyak [R] and Maz'ya and Havin [MH].

5.3. Theorem. Suppose that $1 \leq m<p$ and $E \subset \mathbb{R}^{n}$. Then

$$
\mathcal{H}_{\infty}^{n-m}(E) \leq C \gamma_{p}(E)
$$

where $C=C(n, m, p)$.

Since precise representatives of functions $u \in W^{1, p}\left(\mathbb{R}^{n}\right)$ have Lebesgue points $\mathcal{H}^{n-m}$ almost everywhere [FZ] we may continue as in the proof of Corollary 5.2 to obtain the following.

5.4. Corollary. Suppose that $1 \leq m<p, E \subset \mathbb{R}^{n}, u \in W^{1, p}\left(\mathbb{R}^{n}\right)$ is precisely represented, and that $u \geq 1$ on $E$. Then

$$
\mathcal{H}_{\infty}^{n-m}(E) \leq C \int_{\mathbb{R}^{n}}\left(|\nabla u|^{p}+|u|^{p}\right) d x
$$

Finally, to reach the generality of Theorem 1.3 , we need the following capacitary estimate derived in [FP], [MSZ].

5.5. Theorem. Suppose that $m>1$ is an integer. Let $\mathbf{F}$ be a Young function satisfying (2.2) and (2.3). Let $E \subset \mathbb{R}^{n}$. Suppose that $u \in W_{\mathrm{loc}}^{1,1}\left(\mathbb{R}^{n}\right)$ is precisely represented, $u \geq 1$ on $E$. Then

$$
\mathcal{H}_{\infty}^{n-m}(E) \leq C \int_{\mathbb{R}^{n}}(\mathbf{F}(|\nabla u|)+\mathbf{F}(|u|)) d x .
$$

\section{Applichtion of the general CRiterion}

In this section we prove Theorem 1.3 The reader interested only in the Lebesgue scale of spaces may read the arguments with $\mathbf{F}(t)=t^{p}$ and appeal to Corollary 5.4 This is sufficient to establish Theorems 1.1 and 1.2 To prove the more general result, we use the estimate in Theorem 5.5 
Proof. In view of Theorem 3.3 it is sufficient to verify the assumptions of Theorem 4.4. Select a point $z \in \mathbb{R}^{n+m}$ and $r>0$. Writing $z=\left(x_{0}, y_{0}\right)$ we have

$$
\mathcal{G}_{f} \cap B(z, r) \subset \mathcal{G}_{f} \cap\left[B\left(x_{0}, r\right) \times B\left(y_{0}, r\right)\right] ;
$$

hence,

$$
\pi\left(\mathcal{G}_{f} \cap B(z, r)\right) \subset B\left(x_{0}, r\right) \cap f^{-1}\left(B\left(y_{0}, r\right)\right) .
$$

Let $E=B\left(x_{0}, r\right) \cap f^{-1}\left(B\left(y_{0}, r\right)\right)$ and define $\hat{E}$ by

$$
\hat{E}=\frac{1}{2 r}\left(E-x_{0}\right)=\left\{x \in \mathbb{R}^{n}: x_{0}+2 r x \in E\right\} .
$$

Then

$$
\mathcal{H}_{\infty}^{n-m}(\hat{E})=(2 r)^{m-n} \mathcal{H}_{\infty}^{n-m}(E),
$$

and thus

$$
\mathcal{H}_{\infty}^{n-m}\left(\pi\left(\mathcal{G}_{f} \cap B(z, r)\right)\right) \leq C r^{n-m} \mathcal{H}_{\infty}^{n-m}(\hat{E}),
$$

where $C=C(m, n)$. Now, $\xi \in \hat{E}$ implies that

$$
|\xi| \leq \frac{1}{2} \quad \text { and } \quad \frac{\left|f\left(x_{0}+2 r \xi\right)-f\left(x_{0}\right)\right|}{2 r} \leq \frac{1}{2} .
$$

Thus we consider the test function $u \eta$ where

$$
u(\xi)=2\left(1-\frac{\left.\mid f\left(x_{0}+2 r \xi\right)\right)-f\left(x_{0}\right) \mid}{2 r}\right)^{+}
$$

and $\eta$ is a smooth cutoff function such that $\chi_{B\left(0, \frac{1}{2}\right)} \leq \eta \leq \chi_{B(0,1)}$. If $m=1$, we set $\mathbf{F}(t)=t$ and apply Corollary 5.2 to the function $u \eta$. If $m>1$, we use Proposition 2.1 to find a Young function $\mathbf{F}$ satisfying (2.2) and (2.3) such that

$$
\int_{\Omega} \mathbf{F}(|\nabla u|) d x<\infty
$$

and apply Theorem 5.5 to the function $u \eta$. In either case, we obtain

$$
\begin{aligned}
\mathcal{H}^{n-m}(\hat{E}) & \leq C \int_{B(0,1)}(\mathbf{F}(|u \eta|)+\mathbf{F}(\mid \nabla(u \eta))) d \xi \\
& \leq C \int_{B(0,1) \cap\{u>0\}}(1+\mathbf{F}(|\nabla u|)) d \xi .
\end{aligned}
$$

Applying the change of variable $x \rightarrow x_{0}+2 r \xi$, this becomes

$$
\mathcal{H}^{n-m}(\hat{E}) \leq C r^{-n} \int_{B\left(x_{0}, 2 r\right) \cap f^{-1}\left(B\left(y_{0}, 2 r\right)\right)}(1+\mathbf{F}(|\nabla u|)) d x .
$$

Since

$$
B\left(x_{0}, 2 r\right) \cap f^{-1}\left(B\left(y_{0}, 2 r\right)\right) \subset \pi\left(\mathcal{G}_{f} \cap B(z, 4 r)\right),
$$

(6.1) and (6.2) above imply

$$
\mathcal{H}_{\infty}^{n-m}\left(\pi\left(\mathcal{G}_{f} \cap B(z, r)\right)\right) \leq C r^{-m} \int_{\pi\left(\mathcal{G}_{f} \cap B(z, 4 r)\right)}(1+\mathbf{F}(|\nabla u|)) d x,
$$

verifying the assumptions of Theorem 4.4 with $\theta=C(1+\mathbf{F}(|\nabla u|))$ and thus concluding the proof. 
We now establish a result that yields Theorem 1.1 under a condition that allows some of the coordinate functions to be members of $W^{1, p}\left(\mathbb{R}^{n}\right), p<m$, provided that the remaining ones are Lipschitz. For this purpose, let $1 \leq k<m<n$ and let $h: \mathbb{R}^{n} \rightarrow \mathbb{R}^{m}$ have the form

$$
h=\left(f_{1}, \ldots, f_{k}, g_{k+1}, \ldots, g_{m}\right)
$$

with $f_{1}, \ldots, f_{k} \in W^{1, p}\left(\mathbb{R}^{n}\right), p>k$, and $g_{k+1}, \ldots, g_{m}$ Lipschitz.

6.1. Theorem. With $h$ as above, $|J h| \in L^{1}\left(\mathbb{R}^{n}\right)$ and

$$
\int_{\mathbb{R}^{n}}|J h| d x=\int_{\mathbb{R}^{m}} \mathcal{H}^{n-m}\left(h^{-1}(y)\right) d y
$$

for each measurable set $E \subset \mathbb{R}^{n}$.

Proof. By Theorem 3.3, it suffices to show that $\mathcal{H}^{n}(\bar{f}(N))=0$ whenever $N \subset \mathbb{R}^{n}$ and $\mathcal{L}^{n}(N)=0$. Write $h=(f, g)$ with $f=\left(f_{1}, \ldots, f_{k}\right)$ and $g=\left(g_{k+1}, \ldots, g_{m}\right)$ and let $N \subset \mathbb{R}^{n}$ satisfy $\mathcal{L}^{n}(N)=0$. Identify $\mathbb{R}^{n+k}$ with $\mathbb{R}^{n} \times \mathbb{R}^{k}$ and write $z \in \mathbb{R}^{n+k}$ as $z=\left(z^{\prime}, z^{\prime \prime}\right)$ with $z^{\prime} \in \mathbb{R}^{n}, z^{\prime \prime} \in \mathbb{R}^{k}$. By Theorem 1.2, the set $\bar{f}(N) \subset \mathbb{R}^{n+k}$ has $\mathcal{H}^{n}$ measure zero. Define $g^{*}: \mathbb{R}^{n+k} \rightarrow \mathbb{R}^{n+m}$ by $g^{*}(z)=\left(z, g\left(z^{\prime}\right)\right)$. Then $g^{*}$ is Lipschitz, $h=g^{*} \circ \bar{f}$, and therefore $h(N)=g^{*} \circ \bar{f}(N)$ has $\mathcal{H}^{n}$ measure zero in $\mathbb{R}^{n+m}$.

\section{HÖLDER CONTINUOUS MAPPINGS}

In this section we prove Theorem [1.4. The following result from [HM] plays a crucial role in the proof.

7.1. Theorem. Let $p<n, a>0, \beta \in(0,1)$ and $\gamma>0$. Let $\Omega \subset \mathbb{R}^{n}$ be an open set and $u \in W^{1, m}(\Omega)$ be a nonnegative function such that $u>0$ a.e. Let

$$
Z=\left\{z \in \Omega: \limsup _{r \rightarrow 0} r^{-\beta} f_{B(z, r)} u d x<\gamma\right\} .
$$

Suppose that $\mathcal{H}^{n-m}(Z)>a$. Then there exists a compact set $F \subset \Omega \backslash Z$ such that $\mathcal{L}^{n}(F)>0$ and

$$
\sup _{F} u^{m} \leq C \int_{F}|\nabla u|^{m} d x
$$

where $C=C(n, m, a, \beta)$.

Proof of Theorem 1.4. As in the proof of Theorem 1.1 above, it is sufficient to verify (3.2) whenever $E \subset \mathbb{R}^{n}$ and $\mathcal{L}^{n}(E)=0$. Find an open set $\Omega$ such that $E \subset \Omega$ and

$$
\int_{\Omega}|\nabla f|^{m} d x<\varepsilon
$$

Set

$$
Y=Y_{a}=\left\{y \in \mathbb{R}^{m}: \mathcal{L}^{n}\left(E \cap f^{-1}(y)\right)=0, \mathcal{H}^{n-m}\left(E \cap f^{-1}(y)\right)>a\right\} .
$$

Owing to the Hölder continuity of $f$, given $y \in Y$ we may apply Proposition 7.1 to the set $\Omega$ and the function $u=|f-y|$ and find a $\delta(y)>0$ and a compact set $F(y) \subset \Omega$ such that $\mathcal{L}^{n}(F(y))>0$ and

$$
\sup _{F(y)}|f(x)-y|^{m}<\delta(y) \leq \int_{F(y)}|\nabla f|^{m} d x .
$$


Using a Vitali type covering argument to the system of balls $B(y, \delta(y))$, we find disjointed balls $B\left(y_{j}, \delta_{j}\right)$ and sets $F_{j}$ such that $y_{j} \in Y, \delta_{j}=\delta\left(y_{j}\right), F_{j}=F\left(y_{j}\right)$, and

$$
Y \subset \bigcup_{j} B\left(y_{j}, 5 \delta_{j}\right) .
$$

Since the balls $B_{j}$ are disjointed, the sets $F_{j} \subset f^{-1}\left(B\left(y_{j}, \delta_{j}\right)\right)$ are also disjointed. We infer that

$$
\begin{aligned}
\mathcal{L}^{m}(Y) & \leq \sum_{j} \mathcal{L}^{m}\left(B\left(y_{j}, 5 \delta_{j}\right)\right) \leq C \sum_{j} \delta_{j}^{m} \leq C \sum_{j} \int_{F_{j}}|\nabla f|^{m} d x \\
& \leq C \int_{\Omega}|\nabla f|^{m} d x<C \varepsilon .
\end{aligned}
$$

Letting $\varepsilon \rightarrow 0$ we obtain that $\mathcal{L}^{m}(Y)=0$. Since $a>0$ was arbitrary and the set

$$
\left\{y \in \mathbb{R}^{m}: \mathcal{L}^{n}\left(f^{-1}(y)\right)>0\right\}
$$

is countable (and thus of zero $m$-dimensional measure), we easily conclude the proof.

7.2. Remark. The proof above can be modified to give an independent proof of Theorem[1.1] when $p>m$. Indeed, by [HM] Prop. 3.2], $\mathcal{H}^{n-m}$-a.e. $z \in \mathbb{R}^{n}$ satisfies

$$
\limsup _{r \rightarrow 0} r^{-\beta} f_{B(z, r)}|f(x)-f(z)| d x<\infty
$$

with $\beta=1-\frac{m}{p}$, provided that $f \in W^{1, p}\left(\mathbb{R}^{n} ; \mathbb{R}^{m}\right)$ and $p>m$.

Alternatively, when $p>1$, one can use the fact that for $0<\lambda<1$ and $\varepsilon>0$ there exists an open set $U$ and a mapping $g \in W^{1, p}\left(\mathbb{R}^{n} ; \mathbb{R}^{m}\right)$ so that $g$ is Hölder continuous with exponent $\lambda, f(x)=g(x)$ for all $x \in \mathbb{R}^{n} \backslash U$, and $B_{1-\lambda, p}(U)<\varepsilon$, where $B_{1-\lambda, p}$ is the Bessel capacity. See [BHS], $[\mathrm{S}$; a weaker but also sufficient result of this type is given in [M1. Thus for $f \in W^{1, p}\left(\mathbb{R}^{n} ; \mathbb{R}^{m}\right)$ there is a set $N$ with $B_{1-\lambda, p}(N)=0$ so that (1.2) holds for all $E \subset \mathbb{R}^{n} \backslash N$. In case $p>m$, then $\lambda$ may be chosen so that $\mathcal{H}^{n}(N)=0$, extending (1.2) to all measurable sets $E \subset \mathbb{R}^{n}$.

\section{FURTHER RESUlts ON THE GRAPH MAPPING}

In this section we consider properties of the graph $\mathcal{G}_{f}$ for mappings in the borderline case $W^{1, m}\left(\mathbb{R}^{n} ; \mathbb{R}^{m}\right)$, for $1 \leq m \leq n$. The following theorem was proved in [MM] for $k=n$ and extended to $k \geq n$ in [M4].

8.1. Theorem. Suppose that $k \geq n$ and that $f \in W_{\mathrm{loc}}^{1, n}\left(\mathbb{R}^{n} ; \mathbb{R}^{k}\right)$ is Hölder continuous. Then $f$ satisfies condition $(N)$.

We observe that mappings $f \in W^{1, p}\left(\mathbb{R}^{n} ; \mathbb{R}^{k}\right), p>n$, satisfy the hypothesis of Theorem 3.3 and therefore this result gives a new proof of the area formula of Marcus and Mizel [MMi.

We let $\mathbf{G}(n, m)$ denote the Grassmann manifold of $m$-dimensional subspaces of $\mathbb{R}^{n}$. Given $V \in \mathbf{G}(n, m)$, let $V^{\perp} \in G(n, n-m)$ denote its orthogonal complement. Writing $x \in \mathbb{R}^{n}$ as $x=x_{V}+x_{V^{\perp}}$, with $x_{V} \in V$ and $x_{V^{\perp}} \in V^{\perp}$, the orthogonal projection $P_{V}: \mathbb{R}^{n} \rightarrow V$ is given by $P_{V}(x)=x_{V}$. If $V \in \mathbf{G}(n, m)$ and $W \in \mathbf{G}(n, k)$, $m+k \geq n$, observe that

$$
V^{\perp} \subset W \text { if and only if } W^{\perp} \subset V .
$$


In this case we write $V \perp W$. The following proposition is an immediate consequence of the definitions.

8.2. Proposition. If $V \perp W$, then

1. $P_{V}(W)=V \cap W$,

2. $P_{V}(W+a) \cap P_{V}(W+b)=\emptyset$ for all $a, b \in W^{\perp}, a \neq b$.

Recall that the $n$-dimensional integral-geometric measure of a set $E \subset \mathbb{R}^{n+m}$ is given by

$$
\mathcal{I}^{n}(E)=\int_{\mathbf{G}(n+m, n)} \int_{V} N\left(P_{V}, E, y\right) d \mathcal{H}^{n}(y) d \sigma_{n+m, n}(V),
$$

where $N\left(P_{V}, E, y\right)$ is the number of points $x \in E$ satisfying $P_{V}(x)=y$, and $\sigma_{n+m, n}$ is a normalized Haar measure on $\mathbf{G}(n+m, n)$. In order to prove Theorem 1.5, it suffices to show that $\mathcal{I}^{n}(\bar{f}(E))=0$ whenever $\mathcal{L}^{n}(E)=0$. This is a consequence of the following theorem.

8.3. Theorem. Assume that $1 \leq m \leq n$ and that $f \in W^{1, m}\left(\mathbb{R}^{n} ; \mathbb{R}^{m}\right)$ is Hölder continuous. If $N \subset \mathbb{R}^{n}$ and $\mathcal{L}^{n}(N)=0$, then $\mathcal{H}^{n}\left(P_{V} \circ \bar{f}(N)\right)=0$ for every $V \in \mathbf{G}(n+m, n)$.

Proof. Identify $\mathbb{R}^{n}$ with the subspace $\left\{x \in \mathbb{R}^{n+m}: x_{n+1}=\ldots=x_{n+m}=0\right\}$ of $\mathbb{R}^{n+m}$ and choose $V \in \mathbf{G}(n+m, n)$. Let $W \in \mathbf{G}(n+m, 2 m)$ be any subspace containing $\left(\mathbb{R}^{n}\right)^{\perp}$ and $V^{\perp}$. Then $\mathbb{R}^{n} \perp W$, and hence $W^{\perp} \subset \mathbb{R}^{n}$. Writing

$$
\mathbb{R}^{n}=\left(\mathbb{R}^{n} \cap W\right) \oplus\left(\mathbb{R}^{n} \cap W^{\perp}\right)=\left(\mathbb{R}^{n} \cap W\right) \oplus W^{\perp},
$$

it follows from Proposition 8.2 that the sets $\left\{\mathbb{R}^{n} \cap(W+t): t \in W^{\perp}\right\}$ are disjoint $m$ planes in $\mathbb{R}^{n}$ whose union is $\mathbb{R}^{n}$. Similarly, the sets $\left\{V \cap(W+t): t \in W^{\perp}\right\}$ are disjoint $m$ planes in $V$ whose union is $V$.

For brevity we write $W_{t}=W+t$. Define $h: V \rightarrow W^{\perp}$ by $h=\left.P_{W^{\perp}}\right|_{V}$. Then for $t \in W^{\perp}$, we have $h^{-1}(t)=V \cap W_{t}$. By the co-area formula for Lipschitz mappings between rectifiable sets [F5, 3.2.22], it follows that

$$
\int_{E}\left|J_{n} h\right| d \mathcal{H}^{n}(x)=\int_{W^{\perp}} \mathcal{H}^{m}\left(E \cap W_{t}\right) d \mathcal{H}^{n-m}(t)
$$

for any $\mathcal{H}^{n}$ measurable subset $E$ of $V$, where $J_{n} h$ is the (nonzero constant) $n$ dimensional Jacobian of $h$. Denoting this constant by $J$ and taking $E=P_{V} \circ \bar{f}(A)$ for a Borel set $A \subset \mathbb{R}^{n}$, we have

$$
\begin{aligned}
\mathcal{H}^{n}(E) & =J^{-1} \int_{W^{\perp}} \mathcal{H}^{m}\left(\left(P_{V} \circ \bar{f}(A)\right) \cap W_{t}\right) d \mathcal{H}^{n-m}(t) \\
& =J^{-1} \int_{W^{\perp}} \mathcal{H}^{m}\left(\left(P_{V}\left(\bar{f}(A) \cap W_{t}\right)\right) d \mathcal{H}^{n-m}(t)\right. \\
& \left.\leq J^{-1} \int_{W^{\perp}} \mathcal{H}^{m}\left(\bar{f}(A) \cap W_{t}\right)\right) d \mathcal{H}^{n-m}(t)
\end{aligned}
$$

by Proposition 8.2 and the fact that Hausdorff measure does not increase under orthogonal projection.

On the other hand, since $\bar{f} \in W_{\text {loc }}^{1, m}\left(\mathbb{R}^{n} ; \mathbb{R}^{n+m}\right)$ and $W^{1, m}$ is invariant under any nonsingular linear change of coordinates, Fubini's theorem implies that

$$
\bar{f}_{t}:=\left.\bar{f}\right|_{\mathbb{R}^{n} \cap W_{t}} \in W_{\mathrm{loc}}^{1, m}\left(\mathbb{R}^{n} \cap W_{t} ; \mathbb{R}^{n+m}\right)
$$


for $\mathcal{H}^{n-m}$ almost all $t \in W^{\perp}$. Since $m=\operatorname{dim}\left(\mathbb{R}^{n} \cap W_{t}\right)$, Theorem 8.1 above implies that

$$
\mathcal{H}^{m}\left(\bar{f}_{t}(A)\right)=\int_{A \cap W_{t}}\left|J_{m} \bar{f}_{t}(x)\right| d \mathcal{H}^{m}(x)
$$

for $\mathcal{H}^{n-m}$ almost all $t \in W^{\perp}$ whenever $A \subset \mathbb{R}^{n}$ is Lebesgue measurable and where $J_{m} \bar{f}_{t}$ is the $m$-dimensional Jacobian of $\bar{f}_{t}$. Proposition 8.2 implies that $\bar{f}_{t}(E)=$ $\bar{f}(E) \cap W_{t}$; hence for all such $t$ we have

$$
\mathcal{H}^{m}\left(\bar{f}(A) \cap W_{t}\right)=\int_{A \cap W_{t}}\left|J_{m} \bar{f}_{t}(x)\right| d \mathcal{H}^{m}(x) .
$$

To complete the argument, let $N \subset \mathbb{R}^{n}$ be a set with Lebesgue measure zero and let $A \subset \mathbb{R}^{n}$ be a Borel set containing $N$ with Lebesgue measure zero. Fubini's theorem implies that $\mathcal{H}^{m}\left(A \cap W_{t}\right)=0$ for $\mathcal{H}^{n-m}$ almost all $t \in W^{\perp}$, and therefore

$$
\mathcal{H}^{m}\left(\bar{f}(A) \cap W_{t}\right)=0
$$

for all such $t$. It follows that

$$
\mathcal{H}^{n}\left(P_{V} \circ \bar{f}(N)\right) \leq \mathcal{H}^{n}\left(P_{V} \circ \bar{f}(A)\right) \leq J^{-1} \int_{W^{\perp}} \mathcal{H}^{m}\left(\bar{f}(A) \cap W_{t}\right) d \mathcal{H}^{n-m}(t)=0,
$$

establishing the lemma.

This result is not sufficient to conclude that $\mathcal{H}^{n}(\bar{f}(N))=0$, since there exist sets of positive Hausdorff measure whose projection in every direction has zero measure; cf. [M, Example 9.2]. However, with the help of [M] Corollary 9.8] and [F5, 3.2.27], we arrive at the following.

8.4. Corollary. With $f$ and $N$ as in the proof of Theorem 8.3, $\mathcal{I}^{n}(\bar{f}(N))=0$. In particular, the Hausdorff dimension of $\bar{f}(N)$ does not exceed $n$. Moreover, either $\mathcal{H}^{n}(\bar{f}(N))=0$ or $\bar{f}(N)$ is purely $\mathcal{H}^{n}$ unrectifiable.

\section{REFERENCES}

[AH] Adams, D. R. and Hedberg, L. I., Function Spaces and Potential Theory, Grundlehren der mathematischen Wissenschaften, Springer-Verlag, Berlin, 1996. MR 97j:46024

[BZ] Bagby, T. and Ziemer, W. P., Pointwise differentiability and absolute continuity, Trans. Amer. Math. Soc. 191 (1974), 129-148. MR 49:9219

[BHS] Bojarski, B., Hajłasz, P., and Strzelecki, P., Pointwise inequalities for Sobolev functions revisited, Preprint 2000.

[C] Cesari, L., Sulle trasformazioni continue, Ann. Mat. Pura Appl. 21 (1942), 157-188. MR 6:43c

[E] Eilenberg, S., On $\varphi$ measures, Ann. Soc. Pol. de Math. 17 (1938), 251-252.

[F1] Federer, H., Surface area (II), Trans. Amer. Math. Soc. 55 (1944), 438-456. MR 6:45a

[F2] Federer, H., The $(\phi, k)$ rectifiable subsets of $n$ space, Trans. Amer. Math. Soc. 62 (1947), 114-192. MR 9:231c

[F3] Federer, H., Some integralgeometric theorems, Trans. Amer. Math. Soc. 77 (1954), 238261. MR 16:163b

[F4] Federer, H., Curvature measures, Trans. Amer. Math. Soc. 93 (1959), 418-491. MR 22:961

[F5] Federer, H., Geometric Measure Theory, Grundlehren der mathematischen Wissenschaften, Springer-Verlag, New York, Heidelberg, 1969. MR 41:1976

[Fl] Fleming, W. H., Functions whose partial derivatives are measures, Illinois J. Math. 4 (1960), 452-478. MR 24:A202

[FZ] Federer, H. and Ziemer, W. P., The Lebesgue set of a function whose distribution derivatives are $p$-th power summable, Indiana Univ. Math. J. 22 (1972/73), 139-158. MR 55:8321 
[FM] Fonseca, I. and Malý, J., Remarks on the Determinant in Nonlinear Elasticity and Fracture Mechanics, Applied Nonlinear Analysis, Eds. A. Sequiera, H. B. da Veiga and J. H. Videman, Kluwer Academic / Plenum Publishers, New York, 1999, 117-132. MR 2000k:74010

[FP] Fiorenza, A., and Prignet, A., Orlicz capacities and applications to some existence questions for elliptic PDEs having measure data, preprint.

[H] Hajłasz, P., Sobolev mappings, co-area formula and related topics, Proceedings on Analysis and Geometry, Sobolev Institute Press, Novosibirsk, 2000, 227-254.

[HM] Hencl, S. and Malý, J., Mapping of finite distortion: Hausdorff measure of zero sets, To appear in Math. Ann.

[KKM] Kauhanen, J., Koskela, P. and Malý, J., On functions with derivatives in a Lorentz space, Manuscripta Math. 100 (1999), 87-101. MR 2000j:46064

[K] Kuratowski, K., Topology, Vols. I and II, Academic Press, New York, 1966. MR 36:840, MR 41:4467

[M] Mattila, P., Geometry of Sets and Measures in Euclidean Spaces, Fractals and Rectifiability, Cambridge Studies in Advanced Mathematics 44, Cambridge University Press, 1995. MR 96h:28006

[M1] Malý, J., Hölder type quasicontinuity, Potential Analysis 2 (1993), 249-254. MR 94i:31007

[M2] Malý, J., The area formula for $W^{1, n}$-mappings, Comment. Math. Univ. Carolinae 35 (1994) 291-298. MR 95h:28007

[M3] Malý, J., Absolutely continuous functions of several variables, J. Math. Anal. Appl. 231 (1999) 492-508. MR 2000a:49078

[M4] Malý, J., Sufficient Conditions for Change of Variables in Integral, Proceedings on Analysis and Geometry, Sobolev Institute Press, Novosibirsk (2000) 370-386.

[M5] Malý, J., Wolff potential estimates of superminimizers of Orlicz type Dirichlet integrals, Preprint MATH-KMA-2002/74, Charles University, Praha, 2002.

$[\mathrm{MM}] \quad$ Malý, J. and Martio, O., Lusin's condition (N) and mappings of the class $W^{1, n}$, J. reine angew. Math. 458 (1995) 19-36. MR 95m:26024

[MSZ] Malý, J., Swanson, D., and Ziemer, W. P., Fine behavior of functions with gradients in a Lorentz space, In preparation.

[MMi] Marcus, M. and Mizel, V. J., Transformations by functions in Sobolev spaces and lower semicontinuity for parametric variational problems, Bull. Amer. Math. Soc. 79 no. 4 (1973) 790-795. MR 48:1013

[MH] Maz'ya, V. G. and Havin, V. P., Nonlinear potential theory, Uspekhi Mat. Nauk 27 (1972) 67-138. English translation: Russian Math. Surveys 27 (1972), 71-148. MR 53:13610

[R] Reshetnyak, Yu. G., On the concept of capacity in the theory of functions with generalized derivatives (Russian), Sibirsk. Mat. Zh. 10 (1969) 1109-1138. English translation: Siberian Math. J. 10 (1969), 818-842. MR 43:2234

[RR] Rado, T. and Reichelderfer, P. V., Continuous Transformations in Analysis, SpringerVerlag, Berlin (1955). MR 18:115c

[S] Swanson, D., Pointwise inequalities and approximation in fractional Sobolev spaces, Studia Math. 149 (2002), 147-174.

[VP] Van der Putten, R., On the critical-values lemma and the coarea formula (Italian) Boll. Unione Mat. Ital. Sez. B. (7) 6-B (1992) 561-578. MR 93j:49043

[Z] Ziemer, W. P., Weakly differentiable functions, Graduate Texts in Mathematics 120, Springer-Verlag, New York, 1989. MR 91e:46046

Faculty of Mathematics and Physics, Charles University - KMA, Sokolovská 83, 18675 Praha 8, Czech Republic

E-mail address: maly@karlin.mff.cuni.cz

Department of Mathematics, Texas A\&M University, College Station, Texas 77843

E-mail address: dswanson@math.tamu.edu

Department of Mathematics, Indiana University, Bloomington, Indiana 47405

E-mail address: ziemer@indiana.edu 